\title{
Efficacy of dietary and physical activity intervention in non-alcoholic fatty liver disease: a systematic review
}

\author{
J.B. Moore ${ }^{1,2}$, S Kenneally ${ }^{1}$ and J.H. Sier ${ }^{2}$ \\ ${ }^{1}$ Department of Nutritional Sciences, University of Surrey, Guildford, GU2 7XH, UK and ${ }^{2}$ School of Food Science and \\ Nutrition, University of Leeds, Leeds, West Yorkshire, LS2 9JT, UK
}

Non-alcoholic fatty liver disease (NAFLD) is the most common cause of chronic liver disease worldwide with prevalence above $30 \%$ in many adult populations ${ }^{(1)}$. Strongly associated with obesity, weight loss through diet and physical activity change is the mainstay of its management. This can be difficult to achieve however, and uncertainty exists as to what type of lifestyle changes are most effective $^{(2)}$. Therefore the aim of this work was to systematically evaluate randomised controlled trials assessing diet, exercise or combination interventions aimed at reducing steatosis or markers of NAFLD activity.

The review was conducted by independent reviewers in accordance with PRISMA guidelines and is registered at Prospero (CRD42016032764). Medline, Scopus and Cochrane databases were searched from January $1^{\text {st }} 1980$ through to July $31^{\text {st }} 2016$, for intervention trials assessing the effects of diet, weight loss, exercise or any combination thereof, on NAFLD disease markers in human adults. Risk of publication bias and study quality was assessed using the American Dietetic Association Quality Criteria Checklist.

From a total of 1710 identified records, 24 articles met the inclusion and exclusion criteria; 6 assessed weight loss using dietary restriction, 10 assessed exercise and 8 were combination interventions. Overall, 13 of the 24 studies were rated as high quality trials and those rated as neutral did have a number of positive points. While, all of the trials demonstrated significant reduction in steatosis and/or markers of NAFLD activity, combination interventions appear to be the most effective at improving NAFLD. Results suggest that $5-10 \%$ weight loss using a modestly hypocaloric diet of $500 \mathrm{kcal}$ less per day than calculated energy requirement, in combination with 30-60 minutes exercise on 3-5 days per week should be recommended. The evidence from these interventions show dietary composition is probably less important than total energy intake, and while aerobic and resistance exercise both appear to be equally effective in improving NAFLD, the effect is less so than for weight loss.

We conclude this amount of weight loss is achievable in the trial setting but is challenging in the clinical environment. High intensity, multidisciplinary intervention in specialist clinics is likely to be required in order to manage NAFLD by lifestyle modification alone.

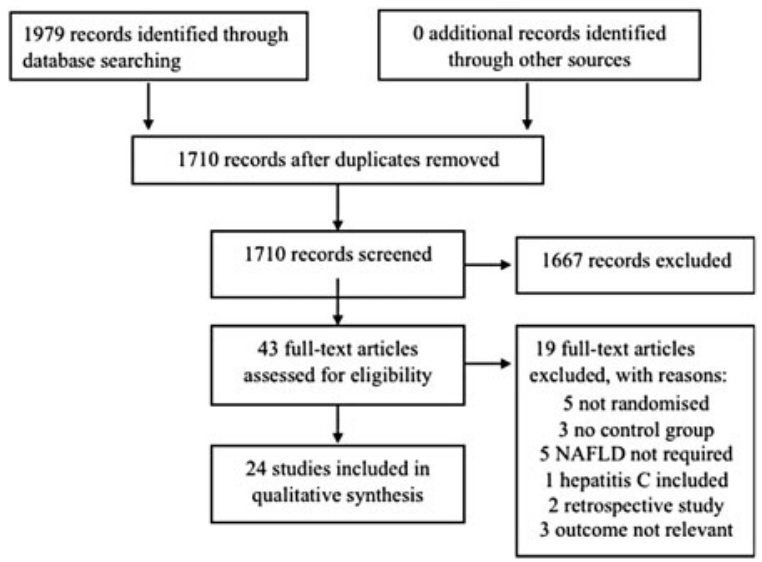

Fig. 1. Flow diagram showing identification and selection of relevant studies for inclusion.

1. Blachier M, Leleu H, Peck-Radosavljevic M et al. (2013) J Hepatol. 58, 593-608.

2. Hannah WN \& Harrison SA (2016) Dig Dis Sci. 61, 1365-74. 